\title{
Group Oriented Traffic Coordination and its Effectiveness
}

\author{
Ankit Sharma \\ M-Tech Scholar, \\ Department of \\ Computer and Science \\ engineering Ajay Kumar \\ Garg Engineering College, \\ Ghaziabad, India
}

\author{
Shashank Sahu \\ Associate Professor, \\ Department of Computer and \\ Science Engineering, \\ Ajay Kumar Garg \\ Engineering College, \\ Ghaziabad, India
}

\author{
Rajesh Kumar Tyagi,PhD \\ Maharaja Surajmal Institute, \\ Delhi, India
}

\begin{abstract}
Rapid urbanization of cities and metro areas has resulted in increasing number of vehicles movement, as a result of that traffic jam tends to form very often at multiple locations.

To find the best effective solution of traffic congestion and the problem of coordination among neighboring vehicles we simulate the group oriented driving approach, vehicles form group with their neighbor vehicle by calculating inclusion function to form a group. This paper presents effectiveness of group oriented traffic approach. Result shows that average speed of vehicles are improved over the random movement of vehicles. Group oriented approach (GOD) is also decreasing fluctuation of average speeds compared to random vehicle movement. The simulation has been run multiple times to find out effectiveness of GOD accurately. We observed achievement of $66 \%$ reduction in fluctuation of speed of cars when they form the group.
\end{abstract}

\section{Keywords}

AOT, A-OTTM, RFID

\section{INTRODUCTION}

The future generation of traffic will be having intelligence system and car to car message transmission capability. This indicates that there is need of latest regulation strategies and migration from hierarchal structure to system having multiple agent structure. Along with this it gives a new path and ideas of using ad-hoc cooperation and coordination of cars and building key components, so as to optimize the productivity and bypass breakdowns. There is a massive increase in the number of vehicles in recent decades; the average number of vehicles passing through a particular is doubled. To deal with this increasing number of vehicles and commuting delay, there should be a proper coordination approach and mechanism that minimizes the travel delay and problem of traffic congestion. Enhancing public transportation is considered as a handful approach on major routes, this includes more number of buses on these routes developing other transport modes and spreading realization of the advantages of cycling and step walking. It is seen as basis for improving traffic congestion as more public transport will be available thereby reducing traffic volume on those routes.

A multi-agent system using fuzzy logic for control of urban traffic system is mentioned in [1]. Improvements in traffic infrastructure is costly affair, such type of projects needs to be precisely evaluated for their effect on traffic. Simulation models using computer can be very useful for making those evaluation in a cost-effective manner. Such models not only evaluate changes under nominal scenarios but also in hypothetical conditions that is quite difficult to perceive in real world case according to Todd Litman [2]. Traffic congestion hampers the lifestyle of many people and needs to be tackled to boost transport facility for everyone according to Shulin He [14]. Referring to this context, we took in to consideration the agent based modeling field and our interest is in simulation of road traffic management. In order to define this model, a driver is taken into consideration as an independent agent whose actions and behavior depends on its environment activities. The resulting generated traffic is from each agent interaction with the regulations, infrastructure of road and other road users. Agent based modeling for simulating traffic is helpful tool for evaluating alternative schemes for traffic engineers as stated by H. M. Zhang [15]. It aims in developing a traffic simulation that helps in understanding traffic theory and assessing network infrastructure and controlling changes. The primary objective is to gain understanding of traffic, learning issues related to traffic simulation, and analyzing agent based modeling as a tool of traffic simulation.

As now a day to deal with the complicatedness in changing distributed systems, such as traffic regulating and managing systems, agent oriented approach is emerging out as a strong computing prototype. Yet various agent-oriented based traffic regulating and managing systems have been recommended and the analysis of multi-agent systems have been done, the applicability of mobile agent applications have not utilized or presented in this field as stated by bon chen [3]. To simulate the model we need platform to run that, so we make use of NetLogo programing language to code our work and various researchers have used this agent simulation platform to model different approaches. A. J. Burns presents the concern about organization security both at micro and macro level and further explaining the use agent based modeling approach in complex adaptive systems and also explained the use of NetLogo simulator in experimenting the model under different scenarios. NetLogo allows researchers to model experience through the communication of agents (called turtles) and the environmental (represented by a grid of cells of that are called patches organized in a coordinate system [4]. Therefore, we present our work using NetLogo simulation platform.

The organization of this paper is as follows. We present the literature survey in section 3 about agent-oriented engineering and multi-agent system in traffic coordination. The solution to the group oriented traffic coordination using Net Logo has been given in proposed work in section 4 . The proposed work has been implemented using Net Logo programming language given in section 5. Final section includes conclusion and future scope. 


\section{LITTERATURE SURVEY}

B. Burmeister et al. 1997 [5], in his work pointed out that Agent-oriented engineering suggests a new way focused on aiding the complete software development operation. All the aspects in the development of software processes are dealt with a single complete thought particularly that of agents, and a system developed by inclusion of agents is known a multiagent system. AOT can be seen as a new advancement in information technology that can aid in responding to the increasing concern in modeling transportation traffic more useful, resource-optimizing and environment-friendly. This article has presented an outline on a varied range of operations where multi-agent systems promises to develop a great effect in this field.

Bo Chen et al. 2010 [6], have given a proposal to integrate mobile agent technology with multi-agent systems to enhance the ability of the traffic management systems to deal with the uncertainty in a dynamic environment. They have refined an IEEE FIPA compliant mobile agent system called Mobile-C and designed an agent-based real-time traffic detection and management system (ABRTTDMS). The system relied on Mobile-C receives benefits of both static and mobile agents. The usage of mobile agents permits ABRTTDMS spontaneously redistributing new regulatory algorithms and works to acknowledge unexpected situations and circumstances. Mobility further diminishes occurrence reaction time and data flow over the network.

ABRTTDMS incorporates varied detection systems that help in enabling traffic regulators to have a detailed outlook of a transit system.

Use of mobile agents for progressive algorithm and operation distribution has been modeled through a laser-based vehicle detection system.

Selvarajah Thuseethan et al. 2015 [7], has given his view about the modern convincing technological improvement in information technology (IT) and also in transmission has given out the exceptionally refined advancements in present systems. Most regular tasks are now programmed with the help of upgraded technologies. Multi-agent systems (MAS) have a significant role in automating the responsibilities in broad range. In this sense current Ocean-Transportation and Traffic Management (OTTM) needs the backbone of artificial intelligence with the help of MAS, conversion and progressive development of ocean-transportation takes us to a well-systemized automated distribution. Current system does not have enough capacity and cannot support the expanding subsequent ocean traffic needs. The suggested Agent based Ocean- Transport and Traffic Management (A-OTTM) approaches are expanded and thoroughly calculated under natural situations with assured condition. With this paper author has tried to present management system based on agent paradigm which is an upcoming Multi-agent A-OTTM simulator.

It permits broad range testing and forecasting of transport and traffic for expected management concepts and outgrowth the capability to analyze new procedures for future oceantransport and traffic control.

Anshul Verma et al. 2015 [8], suggested that as with growing complexities in managing traffic in every domain of real world either in road traffic management, train based management, or in air traffic controlling use of multi-agent technology seems quite interesting. They pointed out the measurement that should be applied to the safety of railway system. The behavioral analysis need to be taken into consideration when designing system for train safety, the scenarios that arise when train run, will be helpful in analyzing the reaction of the system to these conditions.

RFID tag is also helpful for managing and sensing the movement of autonomous vehicles they are being traced and their coordination and cooperation patterns can be used for the behavioral analysis. A study through simulation helps in determining the behavior of the traffic model to achieve moving goal. The work done here is to achieve the objectives that there should be no collision between moving authorities and to analyze the proactive nature of IRMS.

The static parameters are deterministic, the system has advanced knowledge for that, but in case of dynamic parameters they are pointed out and analyzed at runtime this is a challenging task to estimate moving authority.

Jana Gormer et al. 2012 [9], have given their concern about the group-oriented traffic coordination and their approach of forming group in a distributed traffic environment. Group formation of vehicle agents to coordinate either lane on driving or their speed, needs to be evaluated. For smoother traffic movement, giving higher speeds and less time to reach to the target destination, a decentralized approach relying on cooperative driving strategy will prove a favorable approach.

Vehicle agent that are developed with group oriented driving method are capable of perceiving their environment and exchange information often when traffic participants move according to its preferences their movement is restricted by others. Problems of lane switching and fast vehicles are being forced to move slowly because of slow vehicles. The approach described in this is GOD which allows cooperative driving between autonomous vehicle agents. They have the ability to perceive their nearby area, can communicate and can form group to avoid conflict situations.

Decentralized Vehicle Grouping: The main advantage of using decentralized approach is it decreases the overhead of installing extra devices; there is no need of central controller. The centralized approach is limited in communication and computation; it is not possible for a central coordinator to coordinate with all vehicles. The dissimilarity function is one of the criteria of group formation that allow other neighboring members to be included in the group if they satisfy the range of dissimilarity function. Vehicle group $G_{x}$ is defined with leader $\mathrm{x}\left(\mathrm{id}_{\mathrm{x}} ; \mathrm{a}_{\mathrm{x}} ; \mathrm{d}_{\mathrm{x}} ; \mathrm{d}_{\mathrm{sx}}\right)$ as set containing vehicles $\mathrm{y}$ with following property:

$\forall_{\mathrm{y}} \in \mathrm{G}_{\mathrm{x}}, \mathrm{f}(\mathrm{x}, \mathrm{y})<=\alpha_{\mathrm{x}}$

$\alpha_{x}$ is a fixed value predefined by $x$. The attributes $i d_{x} ; a_{x} ; d_{x}$; $\mathrm{d}_{\mathrm{sx}}$ are identifier number, maximal acceleration rate, maximal deceleration rate, and desired speed of $x$. Function $f(x ; y)$ used to calculate the dissimilarity between vehicles $\mathrm{x}$ and $\mathrm{y}$ in terms of their maximal deceleration rate, maximal acceleration rate, and desired speed. Thus, a group leader $x$ will accept any vehicle $\mathrm{y}$ as its member if the dissimilarity $\mathrm{f}(\mathrm{x}$; $y$ ) is smaller than or equals $\alpha_{x}$ Using static attributes as inputs for the dissimilarity function $f(x ; y)$, a group leader $x$ can decide if a vehicle y can participate in its group or not.

GROUP CONFLICTS AND SOLUTION: There might be a situation when group conflicts occur, while group formation that needs to be taken care. The conflict is detected by leader of group. For this the leader or member maintain information about its own group. For finding groups in conflict a group member send information to its neighboring group. By getting 
the response from neighbor a binary function is calculated. Leader regulates its member via group lane.

The paper lacks in pointing out study of application of group life and whether there should be one group or multiple groups, and also coordination architectures and strategies.

Feng chen et al. 2008 [10], according to him:-Urban traffic having high uncertainty, non-linearity in nature and puzzled system. The main aim of traffic control for urban road network is to achieve wide range of coordination for the existing network. Multi-agent system automation is considered as an efficient approach for distributed control. For controlling urban traffic and its coordination, MAS control mechanism is to be used. Cooperation between vehicles on the network communication requirements need to be met out among heterogeneous agent.

Kenneth H Schatten 2013 [11], discussed about sun's massive magnetic fields, the model studies how photosphere environs merge in time. Their solar field depicting procedure uses Net Logo's cellular automata software through algorithm for carrying out the motion of magnetic field over sun through agents. Model entities consist of two breeds: blue and red agents.

ElnazMojahedi et al. 2015 [12], In a Scattered large scale surrounding including interacting entities, simulation dependent on the regional criterions of each network node seems favorable. Agent based modeling and simulation (ABMS) is a more effective modeling paradigm for modeling complex communication networks.

As associates act as active free nodes pertaining individual features, agents are more suitable candidate for hiding them. Therefore they suggested the use of this approach for modeling worm propagation development and conduct their experiments. For doing this they developed a simulation tool using NetLogo environment, which proves to be productive in modeling communication networks.

Juntae Kim et al. 2013 [13], has proposed this research work to establish a link between, information associated to fundamental locations of entities and individuals advice choosing criteria in a friendship or belief network. The implementation of a trust-based transfer system is done in NetLogo, which is a multi-agent open source development tool.

\section{PROPOSED WORK}

The thesis examines the Group Oriented Behavior in order to provide the solution to the problem of traffic management. Group oriented behavior is implemented using Agent Based Programming Language i.e. NetLogo. This language helps in modeling simulator over multiple scenarios that proves useful in carrying out traffic Scenarios that directly cannot be implemented on real-world.
Initially we have taken random number of vehicles then simulation is modified to include them in group and to analyze the behavior of vehicles in Group Oriented Pattern.To achieve and classify results, simulation has been run multiple times each time with different scenarios and parameters into consideration, to find effectiveness of Group Oriented Behavior or traffic management.

After obtaining results from random and group oriented driving simulation, we found out that variance in speed of cars that are not in group has increased considerably to further improve the effectiveness of driving pattern, ungrouping approach has been proposed, and that has given us promising results.

\section{EXPERIMENTATION}

The proposed GOD has been implemented using NetLogo. NetLogo programming language enables us to create as many numbers of agents to perform the simulation on several examples. One of these examples is explained in detail in this Section. In this we have explained the working of the proposed GOD on 6 categories of vehicles for each random vehicles; Group oriented Vehicles and Ungrouped Vehicles.

We have used NetLogo as the programming language for implementing AOT. NetLogo 5.2.0 is used as the platform for writing the code of Group oriented driving using GOD. In the following section

NetLogo and working of the Group oriented driving methodology is explained. Consider a case of random movement of cars each having its own acceleration rate, deceleration rate, and desired speed. Car speed is chosen to be random initially. Result is calculated with random behavior of car X to car Y communication, each car is moving forward with its random speed and the average speed of all the cars, maximum speed and minimum speed is calculated by observing them at different time intervals and the results are calculated when the variance in speed between the cars movement is saturated. In the simulation Red cars are those that are included in the group or that have formed the group and the blue cars are those that does not form the group. Scaling of NetLogo values:

$0=2.5 \mathrm{~km} / \mathrm{hr}, 1=5 \mathrm{~km} / \mathrm{hr}, 2=7.5 \mathrm{~km} / \mathrm{hr}$.

Following tables show the data collected by taking different parameters under different scenarios in NetLogo.

Table 5.1 is showing the numbers of cars that we have used for simulation, next section of table is showing average speed of cars, further section shows minimum speed of random moving cars, maximum speed and the difference between maximum and minimum speed of random moving cars (Blue Cars).

Table 5.1.Table of Random Behavior Analysis Calculating Average Speed

\begin{tabular}{|c|l|l|l|l|l|}
\hline $\begin{array}{l}\text { Number of } \\
\text { cars }\end{array}$ & $\begin{array}{l}\text { Average Speed } \\
\text { of cars }\end{array}$ & Ticks & $\begin{array}{l}\text { Minimum } \\
\text { Speed. }\end{array}$ & $\begin{array}{l}\text { Maximum } \\
\text { Speed }\end{array}$ & $\begin{array}{l}\text { Difference B/W } \\
\text { Max \& Min Speed }\end{array}$ \\
\hline 10 & 0.900 & 200 & 0.388 & 1.615 & 1.227 \\
\hline 20 & 0.950 & 200 & 0.545 & 1.394 & 0.849 \\
\hline 30 & 1.033 & 200 & 0.628 & 1.375 & 0.747 \\
\hline 40 & 1.045 & 200 & 0.729 & 1.237 & 0.508 \\
\hline
\end{tabular}




\begin{tabular}{|c|l|l|l|l|l|}
\hline 50 & 1.08 & 200 & 0.757 & 1.246 & 0.489 \\
\hline 60 & 0.900 & 200 & 0.785 & 1.228 & 0.443 \\
\hline $\begin{array}{l}\text { Average } \\
\text { Speed }\end{array}$ & 0.984 & & & & 0.7105 \\
\hline
\end{tabular}

Random behavior analysis of car has been done and respectively their data has been calculated

Table 5.2 is showing the numbers of cars that we have used for simulation, next section of table is showing average speed of cars, ticks, further section shows minimum speed of cars in group, maximum speed of cars in group, maximum speed of random moving cars, minimum speed of random moving car (Blue Cars) ,difference between maximum and minimum speed of group cars (Red Cars) and the difference between maximum and minimum speed of random moving cars (Blue Cars).

In this table result has been calculated using Net Logo values by considering group oriented approach. In which car will be included in the group if it follows the leader car condition.

Table5.2 Table of Group Oriented Behavior Calculating Average Speed

\begin{tabular}{|l|l|l|l|l|l|l|l|l|l|}
\hline $\begin{array}{l}\text { Number } \\
\text { of cars }\end{array}$ & $\begin{array}{l}\text { Avera- } \\
\text { ge } \\
\text { Speed } \\
\text { of cars } \\
\text { in } \\
\text { group. }\end{array}$ & $\begin{array}{l}\text { Tick } \\
\text { s }\end{array}$ & $\begin{array}{l}\text { Minimum } \\
\text { Speed in } \\
\text { group. }\end{array}$ & $\begin{array}{l}\text { Maximum } \\
\text { Speed in } \\
\text { group. }\end{array}$ & $\begin{array}{l}\text { Average } \\
\text { Speed of } \\
\text { Blue } \\
\text { Cars }\end{array}$ & $\begin{array}{l}\text { Maxim } \\
\text { um } \\
\text { Speed } \\
\text { of Blue } \\
\text { Cars. }\end{array}$ & $\begin{array}{l}\text { Minimu } \\
\text { Speed } \\
\text { Splue } \\
\text { Cars. }\end{array}$ & $\begin{array}{l}\text { Diffrenc } \\
\text { e } \\
\text { B/W } \\
\text { Min } \\
\text { Max. } \\
\text { Red }\end{array}$ & $\begin{array}{l}\text { Diffren } \\
\text { \&e B/W } \\
\text { of } \\
\text { Min \& } \\
\text { Max. } \\
\text { of Blue } \\
\text { cars. }\end{array}$ \\
\hline 10 & 0.968 & 200 & 0.739 & 0.997 & 1.4 & 2.01 & 0.015 & 0.258 & 1.995 \\
\hline 20 & 1 & 220 & 0.991 & 1.131 & 1.211 & 2.02 & 0.015 & 0.240 & 2.005 \\
\hline 30 & 1.11 & 298 & 0.982 & 1.135 & 1.135 & 2.018 & 0.015 & 0.153 & 2.003 \\
\hline 40 & 1.15 & 180 & 1.096 & 1.173 & 1.135 & 2.01 & 0.015 & 0.077 & 1.995 \\
\hline 50 & 1.18 & 32 & 0.845 & 1.20 & 1.211 & 2.01 & 0.015 & 0.128 & 1.995 \\
\hline 60 & 0.937 & 80 & 0.701 & 1.06 & 1.181 & 2.003 & 0.487 & 0.098 & 1.516 \\
\hline $\begin{array}{l}\text { Average } \\
\text { Speed }\end{array}$ & 1.057 & & & & & & & 0.159 & 1.918 \\
\hline
\end{tabular}

Figure 5.1 Random behavior has been presented that shows fluctuation of speeds of cars moving in a random fashion, they are not forming any group.

Figure 5.2 Simulation result shows that group oriented behavior effectiveness in which all vehicles (Red cars) that form the group are moving with a constant average speed after sometime of simulation run. Average speed of grouped vehicles increases gradually.

Figure 5.3 Shows the need of ungrouping after sometime, when a suitable group has been formed. Vehicles which are not part of the group showing very high fluctuation in their speed therefore ungrouping behavior is imposed now and some vehicles are removed from the group therefore it has a mixed combination of vehicles under group and without group, graph represents average speed of both category i.e. grouped and ungrouped vehicles in which it is showing less fluctuation in their speed.

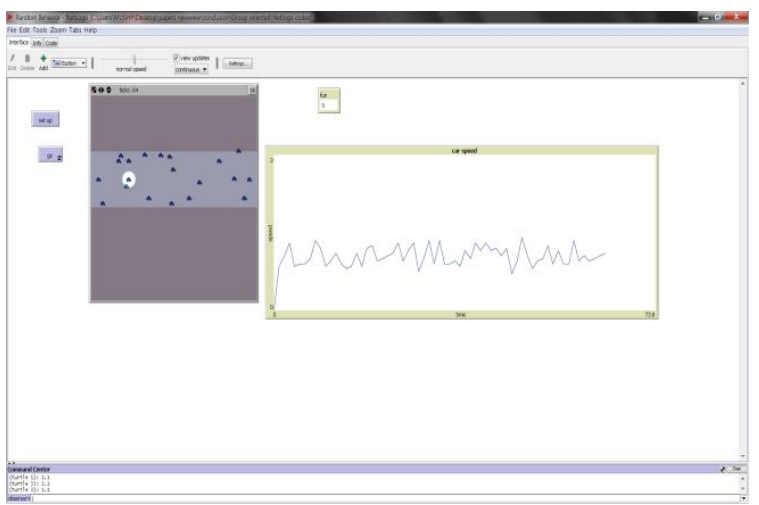

Figure 5.1 Random Behavior of Cars

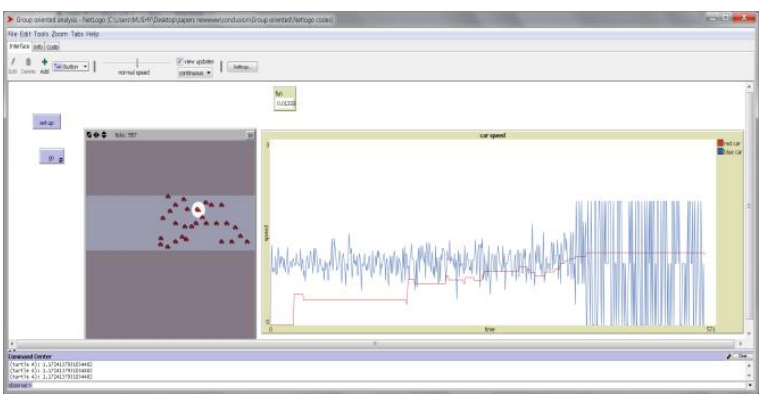

Figure 5.2 Group Oriented Behavior 


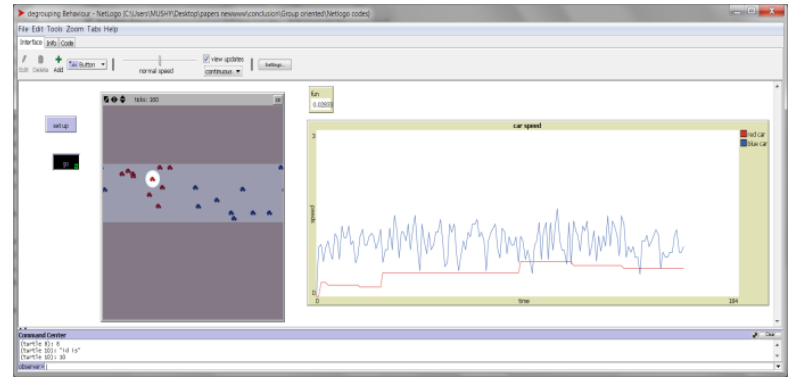

Figure 5.3 Ungrouped Behaviors

\section{CONCLUSION AND FUTURE SCOPE}

Managing traffic in a rapidly growing cities and countries is a challenging task and needs to be addressed effectively and efficiently. Lack of coordination and communication among vehicles moving on the road is the main hurdle in the advancement of traffic movement. There is a need to build advanced traffic management strategy and technology that uses agent technology to manage and coordinate traffic flow, agents have the ability to sense and adapt themselves accordingly to the environment in which they are deployed. Group oriented driving can be seen as a favorable approach to coordinate vehicle movement on roads, it allows vehicles to form group and move together with the speed of group leader. In our work we have given detailed description of Group oriented driving and benefits of including other vehicles in the group thereby achieving improved average speed of grouped vehicles. Simulation result has been gathered under different scenarios by considering different number of vehicles each time, results are calculated using random number of vehicles, grouped vehicles and also through ungrouped vehicles. Our results of removing vehicles from the group also show that the randomness in the speed of the cars that were not in the group reduced considerably. Future work will analyze and correlate various group architecture of coordination and scenario for calculating group life time and blueprints of initiating and discarding groups. We propose to explore richer car models inheriting planned learning, and considering agents interaction and infrastructural elements. Further area of research of ours would be communication, width of road, considering other vehicles other than cars.

\section{REFERENCES}

[1]. Jamshidnejad, Anahita, Bart De Schutter, and Mohammad J. Mahjoob. "Urban traffic control using a fuzzy multi-agent system." Control Conference (ECC), 2015 European. IEEE, 2015.

[2]. Todd Litman, "Smart Congestion Relief: Comprehensive Analysis Of Traffic Congestion Costs and Congestion Reduction Benefits." (2011).

[3]. Bo Chen, Harry H. Cheng, and Joe Palen. "Integrating mobile agent technology with multi-agent systems for distributed traffic detection and management systems." Transportation Research Part C: Emerging Technologies 17.1 (2009).
[4]. Burns, A. J., et al. "Organizational information security as a complex adaptive system: insights from three agentbased models." Information Systems Frontiers (2015).

[5]. Burmeister, Birgit, Afsaneh Haddadi, and Guido Matylis. "Application of multi-agent systems in traffic and transportation." Software Engineering. IEE Proceedings. Vol. 144. No. 1. IET, 1997.

[6]. Bo Chen, and Harry H. Cheng. "A review of the applications of agent technology in traffic and transportation systems." Intelligent Transportation Systems, IEEE Transactions on 11.2 (2010): 485-497.

[7]. SelvarajahThuseethan, and Shanmuganathan Vasanthapriyan. "Multi-agent based ocean-transport and traffic controlling system: A simulation." Information Technology: Towards New Smart World (NSITNSW), 2015 5th National Symposium on. IEEE, 2015.

[8]. Anshul Verma, and K. K. Pattanaik. "Multi-agent communication-based train control system for Indian railways: the behavioral analysis." Journal of Modern Transportation 23.4 (2015).

[9]. Jana Görmer, and Jörg P. Müller. "Multiagent system architecture and method for group-oriented traffic coordination." Digital Ecosystems Technologies (DEST), 2012 6th IEEE International Conference on. IEEE, 2012.

[10].Feng Chen and Hao Pang, "Study of Multi-agent Area Coordination Control for Urban Traffic", Proceedings of the $7^{\text {th }}$ World Congress on Intelligent Control and Automation June 25 - 27, 2008, Chongqing, China.

[11].Kenneth H Schatten "Large-scale solar magnetic field mapping”, Springer Plus 2013.

[12].ElnazMojahedi, and Mohammad Abdollahi Azgomi. "Modeling the propagation of topology-aware P2P worms considering temporal parameters." Peer-to-Peer Networking and Applications 8.1 (2015):Springer Science.

[13].Juntae Kim and Somayeh. "Using structural information for distributed recommendation in a social network." Applied intelligence 38.2 (2013): 255-266.

[14].He, Shulin. "Quantitative Problem of Road Traffic Congestion Simulation and Extension Information Analysis." International Journal of Emerging Technology and Advanced Engineering (ISSN 2250-2459, Volume 2, Issue 2, February 2012.).

[15].Zhang, H., Jingtao $\mathrm{Ma}$, and $\mathrm{Yu}$ Nie. "Local synchronization control scheme for congested interchange areas in freeway corridor." Transportation Research Record: Journal of the Transportation Research Board 2128,(2009):173-183. 